\title{
Author Index Vol. 34, 1994
}

Abbruzzese, M. 135 Ajmar, F. 135 Alberoni, M. 324 Albers, J.W. 173 Angelini, C. 95 Anlar, B. 44 Arai, H. Ill Azzimondi, G. 332

Baba, M. 221 Babinsky, R. 290 Barkhof, F. 83 Batocchi, A.P. 78 Becker, J.T. 277 Bellone, E. 135 Berciano, J. 344 Bernardi, G. 316 Bertolasi, L. 107 Bertora, P. 341 Bezerianos, A. 268 Bird, T.D. 155 Bischof, F. 263 Blaivas, M. 173 Bogousslavsky, J. 64, 306 Bracchi, M. 87 Bradac, G.B. 87 Bressi, S. 324 Bridger, J. 295 Bromfield, E.B. 186 Buttner.T. 162

Caccia, M.R. 341 Calabrese, P. 290 Calne, D.B. 1 Canal, N. 324 Caplan, L.R. 53,114,186 Caskey, C.T. 181 Casmiro, M. 292 Ceccaldi, M. 36 Chambron, J. 224 Chaudhuri, K.R. 295 Chen, C.C. 16 Chiba, K. 140 Chopard, J.-L. 64 Clemens, P.R. 181 Combarros, O. 344 Comi, G. 324 Cortes, J. 193 Costa, B. 107 Crabtree, L.D. 155 Crepin-Leblond, T. 64 Cupini, L.M. 316

D’Alessandro, R. 332 Daniele, D. 87 DeKosky, S.T. 277 Dew, M. A. 277 Dufour, A. 199

Eishi, Y. 298 Ellis, S. 147 Eoli, M. 199 Escartin, A. 209 Estade, M. 283 Eto, H. 286 Evoli, A. 78

Felice, K. J. 173 Fiaschi, A. 107 Filippi, M. 324 Fischer, E.G. 114 Fish, D. 320 Flaburiari, K. 268 Frackowiak, R.S.J. 295 Fuh, J.L. 16 Fukui, T. 158,272 Fumoleau, P. 236 Furukawa, T. 110,298 Fusi, L. 295 Gamondes, P. 288 Garcia-Monco, J.C., 193 Gehlen, W. 290 Goadsby, P.J. 30 Godefroy,0. 11 Gomez Beldarrain, M. 193

Hamann, G.F. 99 Hantson, P. 113 Hartard, C. 337 Heye, N. 162 Hirsch, N.P. 320 Howard, R.S. 320 Hu, H.H. 16

Ichikawa, H. 158 Imir, T. 44 Irie, H. 253 Isenberg, E. 99 Ishii, A. 177 Isogai, Y. 23

Jager, R. 295 Janetzky, B. 263 Jennum, P. 204 Jost, W.H. 329

Ju, T.H. 16

Jung, G. 329 Jiirgens, U. 213

Kameda, N. 110 Kaneko, E. 110 Kao, K.-P. 103 Kasai, H. 158 Kato, A. 298 Kaube, H. 30 Kelly, J. J., Jr. 114 Keshevar, T. 99 Kesselring, J. 300 Kohler, A. 283 Konstantinou, D. 268 Koudstaal, P.J. 61 Kuhn, W. 263 Kulisevsky, J. 209 Kumazawa, K. 221 Kuntzer, T. 4 Kunze, K. 337

LahLR. 213 LaMantia, L. 199 Lang, C. 113 LaVecchia, C. 301 Lazzarino, L.G. 230 Leppik, I.E. 186 Levi, F. 301 Leys, D. 11,83 Liao, K.K. 16 Lindeneiner, N. v. 40 Lipe, H.P. 155 Llorens, V. 193 Loire, R. 288 Loiseau, P. 4 Lopez, O.L. 277 Lorusso, S. 292 Louboutin, J.P. 236

Magistris, M.R. 283 Maier, B. 290 Mancardi, G.L. 135 Mandich, P. 135 Mangoni, A. 341 Marchau, M., Jr. 11 Markowitsch, H.J. 290 Marti-Fabregas, J. 209 Martinelli, V. 324 Maruyama, S. 168 Matteis, M. 316 Maugard-Louboutin, C. 236 Mauss, Y. 224

May, T.W. 213 Mayo, J. 193 Mendoza, G. 209 Michikawa, M. 110 Milandre, L. 36 Milanese, C. 199 Miller, D.H. 320 Miro, J. 344 Mitsuma, T. 221 Miyazaki, T. 221 Mizutani, T. 253 Mochio, S. 23 Mochizuki, A. 286 Mohr, M. 224 Moili, R. 99 Mori, E. 243 Moseley, I.F. 320 Moser, H.W. 241 Moulin, T. 64 Mounier-Vehier, F. 11

Namer, IJ. 224 Negishi, A. 158 Nicolai, A. 230 Nighoghossian, N. 288 Nishiyama, K. 48

O’Donoghue, M. 4 Ohkoshi, N. 177 Oka, H. 23 Owada, K. 298 Ozawa, E. 253

Pagni, C.A. 87 Palmisani, M.T. 78 Papadakis, N. 268 Papapetropoulos, T. 268 Papathanasopoulos, P. 268 Pareyson, D. 135 Pazzaglia, P. 332 Perrin, L. 288 Pierrot-Deseilligny, C. 121 Poli, V. 292 Poncet, M. 36 Poole, R.M. 173 Porchet, F. 114 Poulet, P. 224 
Provenzano, C. 78 Pruvo, J.P. 11 Przuntek, H. 162,263

Rambeck, B. 213 Reichmann, H. 40,95,263 Riccio, A. 87 Richens, A. 4 Rinaldi, R. 332 Riva, A. 87 Rondepierre, Ph. 11 Rouyer, C. 36

Saito, F. 253 Saitoh, T. 221 Sakamaki, T. Ill Sakuta, M. 48 Salmaggi, A. 199 Samkoff, L.M. 228 Sander, J.WA.S. 186 Sasaki, K. 140 Sato, K. 23 Sato, Y. 272 Scheltens, P. 83 Schenone, A. 135 Schimrigk, K.

99,329 SchnabeLR. 213 Schoji, S. 286 Schols, L. 263 SeibeLP. 263 Sghirlanzoni, A. 135 Shoji, S. 177 Silvestrini, M. 316 Sirabian, G. 324 SJ0l, A. 204 Small, M. 147 Smith, C.R. 228 Sobue, G. 221 Soma, Y.

168 Somsak, D. 277 Steibel, J. 224 Steinling, M. 83 Stoll, M. 99 Stracciari, A. 332 Strittmatter,|M. 99 Stura, G 87 Sugita, K. 158,272 Suzuki, H. 140 
Tachibana, N. 320 Takahashi, H. 140 Takasu, M. 286 Takeda, K. 48 Takeuchi, T. 272 Tamura, K. 140 Tei, H. 168 Thomas, D.G.T. 295 Tohgi, H. 140 Tonali, P. 78 Torri, V. 199 Tribolet, N. de 114 Trillet, V. 288 Troisi, E. 316 Trouillas, P. 288 Tsai, C.-P. 103 Tsuchiya, K. 253 Tsukagoshi, H. 158,272 Turanh, G. 44

Uccelli, A. 135 Ugawa, Y. 48 Uitti, RJ. 1 Utsunomiya, K. 286

Wermersch, PJ. 16

Yalaz, K. 44 Yamada, M. 110,298 Yamadori, A. 243 Yamashita, H. 243 Yamawaki, M. 110 Yanagi, T. 221 Yasuda, T. 221 Yen, DJ. 16 Yokota, T. 298 Yoneda, Y. 243 Zaccari, G. 316 Zanette, G. 107

350 
